\title{
VLBI at the highest frequencies - AGN studied with micro-arcsecond resolution
}

\section{Thomas P. Krichbaum*}

Max-Planck-Institute für Radioastronomie, Bonn, Germany

E-mail: tkrichbaumempifr-bonn.mpg.de

\section{Agudo}

Max-Planck-Institute für Radioastronomie, Bonn, Germany

E-mail: iagudo@mpifr-bonn.mpg.de

\section{U. Bach}

Max-Planck-Institute für Radioastronomie, Bonn, Germany E-mail: ubachempifr-bonn.mpg.de

\section{A. Witzel}

Max-Planck-Institute für Radioastronomie, Bonn, Germany E-mail: awitzel@mpifr-bonn.mpg.de

\section{J. A. Zensus}

Max-Planck-Institute für Radioastronomie, Bonn, Germany

E-mail: azensus@mpifr-bonn.mpg.de

\begin{abstract}
Compact galactic and extragalactic radio sources can be imaged with an unsurpassed angular resolution of a few ten micro-arcseconds, adopting the observing technique of global millimeter VLBI. Here, we present the Global Millimeter VLBI Array (GMVA) and discuss its present performance. We show and discuss new results obtained with the GMVA for individual and partially archetypal radio sources with prominent VLBI jets (e.g. 3C 120, Cygnus A, M 87, 3C 454.3, NRAO150). The variety of observed effects range from jet propagation and bending, partial foreground absorption in the nucleus, and jet component ejection after major flares to new and very small (15-20 Schwarzschild radii) upper limits to the jet base of M 87. We also discuss the future development of mm-VLBI at $3 \mathrm{~mm}$ and towards shorter wavelengths, and make suggestions for possible improvements.
\end{abstract}

The 8th European VLBI Network Symposium

September 26-29, 2006

Toruń, Poland

\footnotetext{
*Speaker.
} 


\section{Introduction}

VLBI studies at centimeter wavelengths have already revealed a wealth of details about the morphology and propagation of the powerful AGN radio jets. The detailed understanding of their physical origin, however, is still limited and it is very unclear, how these jets are launched and accelerated and how the jet base is connected to the 'central engine'. The recent development in black hole theory and computer simulations suggest that general relativistic magneto-hydrodynamical (GR-MHD) processes, i.e., the interplay of matter accretion, magnetic fields and space-time curvature, eventually modified by black hole rotation (Kerr metric), may play an important role (see [15], [10], [19]). However, not many observational constraints to the physical parameters of these models are yet available. It is also unclear, if GR-MHD-driven jet acceleration occurs in all classes of radio-loud AGN, or is present in only some objects. It is hoped that VLBI studies of AGN at short millimeter wavelengths ( $\lambda \leq 3 \mathrm{~mm}, \mathrm{~mm}-\mathrm{VLBI}$ ) can contribute to answering these questions. Depending on the physical details of the jet acceleration and collimation processes, the compactness and brightness temperatures of the radio cores must change with distance from the center. Similarly, the morphological, spectral and kinematical properties of the innermost jet regions will change, depending on the nature of the 'central accelerator'. Therefore, VLBI imaging at the highest possible frequencies should be used to determine/measure the variation of observational parameters, like i.e., the brightness temperature, jet velocity and jet spectrum along the jet, and as close as possible down to the jet origin. Since at $\mathrm{cm}$-wavelengths the jet base appears self-absorbed and unresolved, the highest possible observing frequencies and angular resolutions are required to image and study these regions.

\section{Present-day Global Millimeter VLBI}

We will now focus on some new results obtained with the Global mm-VLBI Array (GMVA) ${ }^{1}$. The GMVA is open to the scientific community, offering global VLBI observing in the 3-mm band on a regular basis. At present, two observing sessions per year, each of up to 5 days duration, are carried out. More frequent observations are in principle possible, if the demand for this (via proposal pressure) increases. Proposal calls are synchronized with the annual February 1st and October 1st NRAO deadlines. The GMVA combines the European antennas (the 100-m Effelsberg (EB), Germany, the 30-m Pico Veleta (PV), Spain, the phased $6 \times 15-\mathrm{m}$ Plateau de Bure Interferometer (PdB), France, the 20-m Onsala (ON), Sweden, and the 14-m Metsähovi (MET), Finland) with the VLBA, of which eight antennas are presently equipped with 3-mm receivers.

In Figure 1, we show some typical $u v$-coverages for the GMVA for sources at four different, representative declinations. Mainly due to the participation of the two very sensitive IRAM telescopes (PV \& PdB) and the 100-m Effelsberg telescope, the array sensitivity is improved by a factor of 3-4, when compared to the VLBA alone. In the standard observing mode with Mark 5 recording at $512 \mathrm{Mbit} / \mathrm{s}$, the single baseline $7 \sigma$-detection threshold on the most sensitive PV-PdB baseline is $30-40 \mathrm{mJy}$, and from the IRAM antennas to one of the VLBA antennas it is 50-100 mJy (assuming $100 \mathrm{~s}$ integration and $20 \mathrm{~s}$ coherence time). This has to be compared with a $200-300 \mathrm{mJy}$ detection level, which is reached between two VLBA antennas. The intra-American sensitivity will improve,

\footnotetext{
${ }^{1}$ web link: http://www.mpifr-bonn.mpg.de/div/vlbi/globalmm
} 

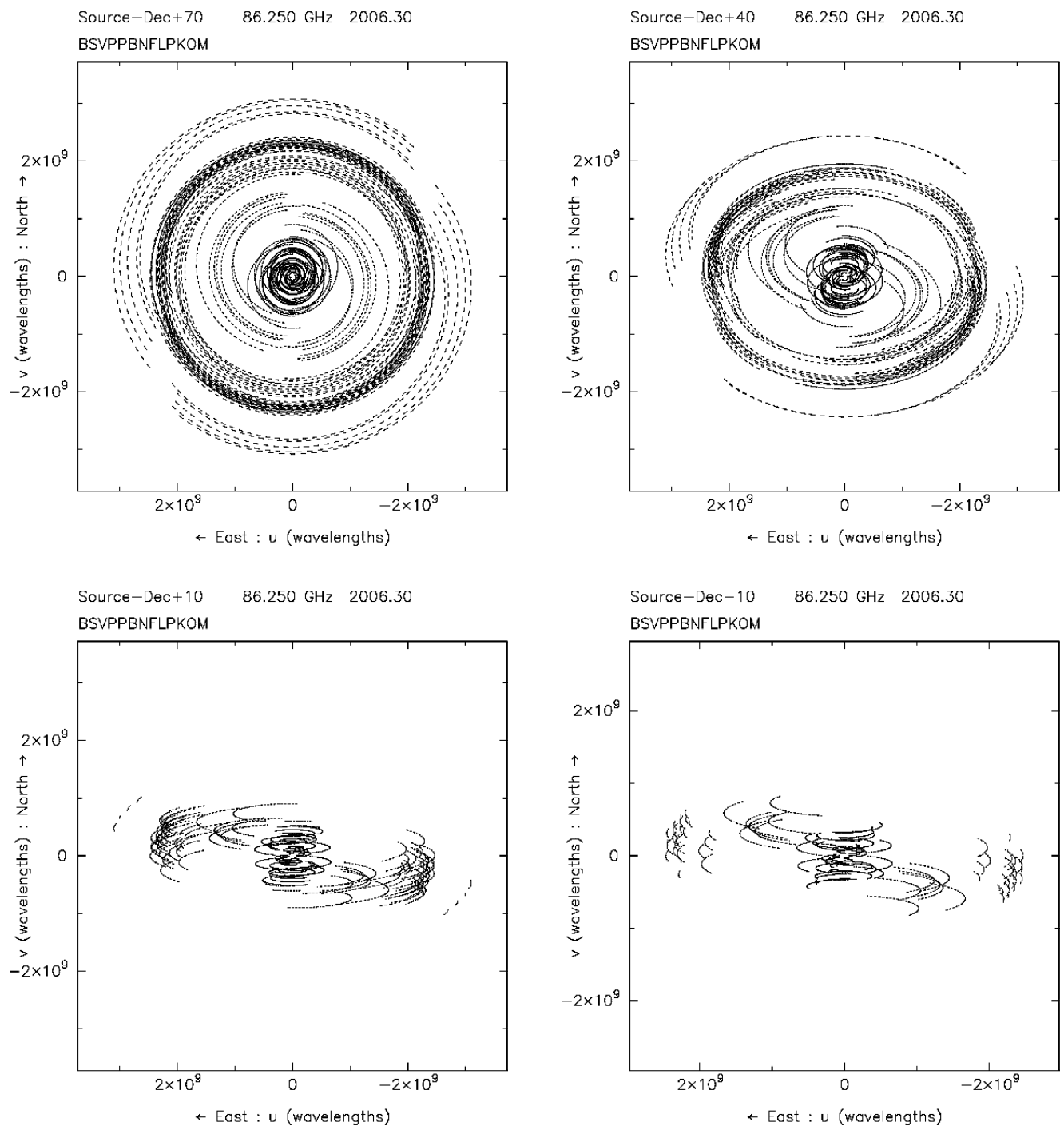

Figure 1: Typical $u v$-coverages for the VLBI array of the 13 radio telescopes presently participating in the global 3-mm VLBI with the GMVA. For each observation, a duration of $12 \mathrm{~h}$ and a duty cycle (VLBI time over total time) of $\sim 50 \%$ was adopted. >From top left to bottom right, $u v$-coverages for a source at declination $\delta=70^{\circ}, 40^{\circ}, 10^{\circ}$, and $-10^{\circ}$ are shown.

when the 100-m Green Bank telescope (GBT) becomes available for 3-mm VLBI. The quality of present-day 3-mm VLBI maps and their rms noise level is mainly determined by the atmospheric conditions during the observations. As a compromise for good observing conditions at all VLBI stations, observations in spring (April, May) and autumn (October) are performed. It is frequently discussed if mm-VLBI observations during winter time (January, February) might be better suited. However, cold periods of low humidity appear often only locally and are neither well predictable nor long enough in present European climate. With up to 13 stations participating in a GMVA observation, images with a dynamic range of up to a few hundred can be obtained, reaching a rms noise level of $\sim 0.5-2 \mathrm{mJy} / \mathrm{beam}$ for a 12-hour full $u v$-coverage observation (at $512 \mathrm{Mbit} / \mathrm{s}, 50 \%$ duty cycle). In mm-VLBI the short coherence time and the atmospheric opacity variations require 
the heavy use of closure amplitudes and amplitude self-calibration down to the time scale of atmospheric fluctuations. Therefore it is vital for mm-VLBI that the number of participating telescopes is as large as possible $(N \geq 10)$.

For compact galactic and extragalactic radio sources, the GMVA provides VLBI images with an angular resolution of up to $40 \mu$ as. For a source at redshift $z=0.1$, this corresponds to a linear scale of $2.2 \cdot 10^{17} \mathrm{~cm}$ (or $0.07 \mathrm{pc}$ or 85 light days) ${ }^{2}$. When expressed in terms of Schwarzschild radii of a black hole of mass $10^{9} \mathrm{M}_{\odot}\left(R_{S}^{9}:=R_{S}\left(10^{9} \mathrm{M}_{\odot}\right)\right)$, a linear size of $740 R_{S}^{9}$ is obtained, comparable to the expected size of an accretion disk. Mainly technically-driven VLBI pilot experiments, performed at $2 \mathrm{~mm}$ and $1 \mathrm{~mm}$ have recently revealed first transatlantic fringes at a world record fringe

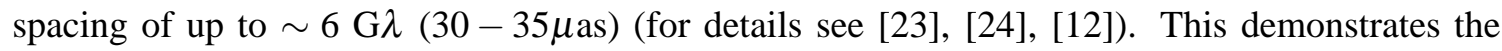
principal feasibility of global VLBI at wavelengths shorter than $\lambda \leq 2 \mathrm{~mm}$ and shows that VLBI imaging with $15-20 \mu$ as resolution is within reach. Depending on source distance and central black hole mass, this corresponds to sizes ranging from a few to a few hundred gravitational radii (or Schwarzschild radii). This resolution is so high that future and more sensitive mm-VLBI experiments at $\lambda \leq 1 \mathrm{~mm}$, should facilitate the observation of direct signatures of General Relativity (GR) effects near super massive black holes (SMBH).

\subsection{Examples of radio jets studied with 3-mm VLBI:}

In the following section we highlight some new and partly still preliminary results obtained from recent global 3-mm VLBI observations. The results of a more statistically oriented new 3-mm VLBI detection survey are presented by Lee et al. (this conference).

3C 120: In Figure 2 , we show two VLBI maps of 3C 120 obtained with the GMVA in April 2003 (10 stations, mode: 256-8-2, $11 \mathrm{~h}$ ) and in October 2004 (12 stations, mode: 512-8-2, $12 \mathrm{~h}$ ). The dynamic ranges of the two maps are 200:1 and 300:1, respectively. At the redshift of $z=0.033$, the observing beam size of $\sim 0.25 \times 0.06$ mas corresponds to a spatial resolution of $0.16 \times 0.04 \mathrm{pc}$, or in terms of a $3 \cdot 10^{7} M_{\odot}$ black hole to a linear scale of $4.1 \times 10^{4} R_{S}^{7}$ for the minor beam axis. At and below the 0.5 mas scale, structural differences and a misalignment of the orientation of the inner jet between both epochs are visible. Much more densely time-sampled observations will be necessary to follow the expected motion of $\sim 2$ mas/yr ([17]). A slightly super-resolved map of April 2003, reveals an 'S-shaped' structure on the $0.1-0.2$ mas scale. This and the change of the position angle of the sub-mas jet on a 1.5-year time scale support the idea of helical Kelvin-Helmholtz instabilities that may be induced by some sort of 'rotation' at the jet base ([9], [18]).

Beyond the 0.5 mas scale, the 3-mm-VLBI jet fades and breaks into filamentary sub-components. A demonstration of the reality of this weak and extended jet emission is obtained from the inspection of the right panel of Figure 2 . Here, we show $u v$-tapered VLBI images observed within a 16-day interval at $43 \mathrm{GHz}$ (VLBA) and $86 \mathrm{GHz}$ (GMVA). Both maps are convolved with the same CLEAN-beam of size $0.3 \times 0.1$ mas and show weak jet emission at corresponding locations on the mas-scale. Future, more detailed analysis will be necessary to find out if the partial misalignment of the jet ridge-line in $0.2-0.5$ mas region can be interpreted by transverse opacity effects and jet

\footnotetext{
${ }^{2}$ Through out this paper we adopt the following cosmological constants: $H_{0}=71 \mathrm{~km} / \mathrm{s} / \mathrm{Mpc}, \Omega_{m}=0.27, \Omega_{\Lambda}=0.73$.
} 


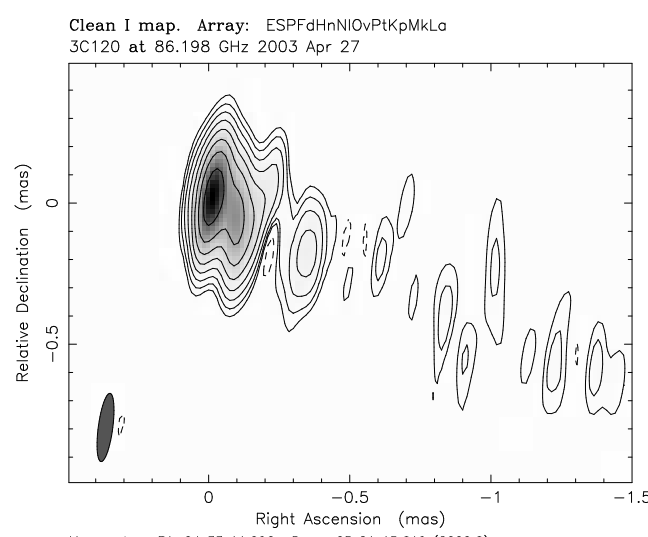

Map center: RA: 043311.096 , Dec: +052115.619 (2000.0) Mapp peak: $0.845 \mathrm{Jy} /$ beam
Contours \%: -0.50 .51248163264
Bearm FWHM: $0.244 \times 0.0514$ (mas) ot -7.530

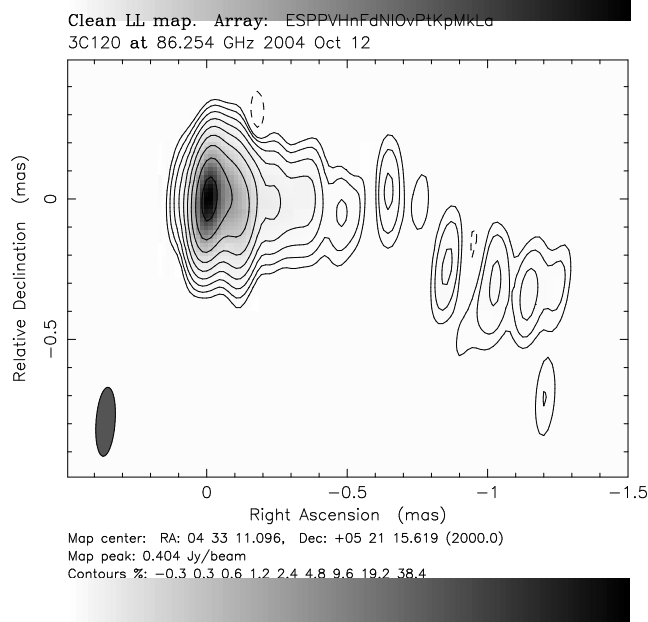

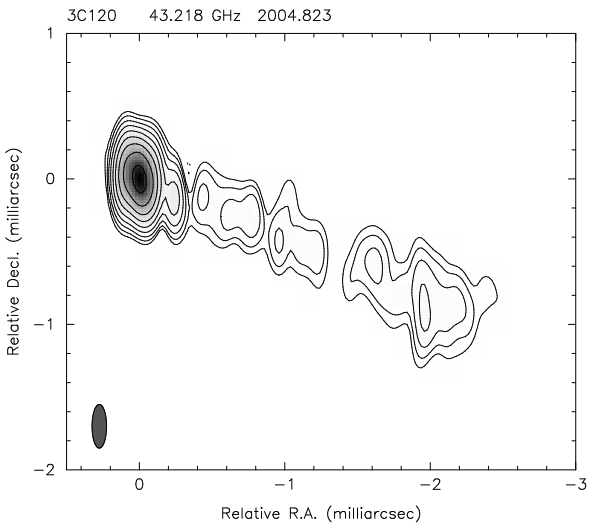

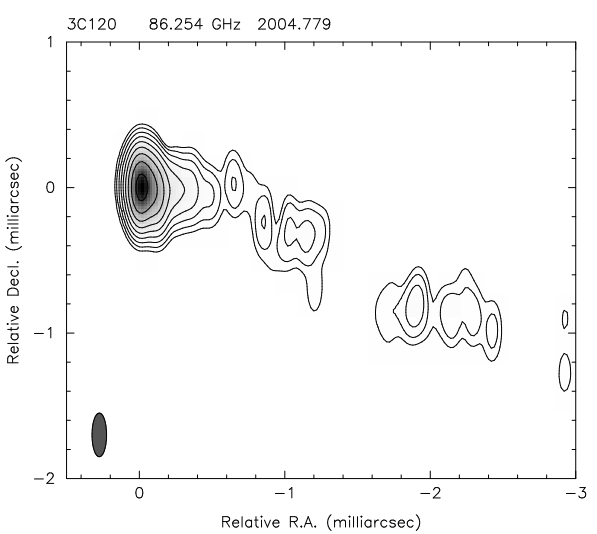

Figure 2: Left: Clean maps of 3C 120 observed at $86 \mathrm{GHz}$ with the GMVA in April 2003 (top) and October 2004 (below). Contours and peak fluxes are shown below each map. Right: 3C 120 observed at $43 \mathrm{GHz}$ with the VLBA (top) and at $86 \mathrm{GHz}$ with the GMVA (below). The $86-\mathrm{GHz}$ image is from October 12, the 43-GHz image is from October 28, 2004 (Marscher et al., [28]). Contour levels are at -0.3, 0.3, 0.6, 1.2, 2.4, 4.8, 9.6, $19.2,38.4$, and $76.8 \%$ of peak flux densities of $0.73 \mathrm{Jy} / \mathrm{beam}$ at $43 \mathrm{GHz}$ and $0.46 \mathrm{Jy} / \mathrm{beam}$ at $86 \mathrm{GHz}$. For a better comparison, both maps are convolved with the same elliptical beam of size $0.3 \times 0.1$ mas.

stratification.

M 87: The nearby radio galaxy M 87 (Virgo A, 3C 274) is one of the closest $(z=0.00436, D=$ $16.75 \mathrm{Mpc}$ ) radio galaxies with a prominent radio jet. It is an ideal object for high spatial resolution VLBI studies that address the question how the powerful and relativistic jets in AGN are formed. For the assumed black hole mass of $3 \cdot 10^{9} M_{\odot}$, the Schwarzschild radius is $R_{S}^{9}=8.9 \cdot 10^{14} \mathrm{~cm}$, which translates for a GMVA observing beam of $50-60 \mu$ as to an exceptionally high spatial resolution corresponding of only $14-17$ Schwarzschild radii for this source.

We note that a similar spatial resolution in terms of Schwarzschild radii is obtained for the $3.6 \cdot 10^{6} M_{\odot}$ black hole in the center of our Galaxy $\left(\mathrm{Sgr} \mathrm{A}^{*}\right)$ at a shorter wavelength of $1.3 \mathrm{~mm}$ (for details see [22], [25] and references therein). The low declination of Sgr A* and the lack of 

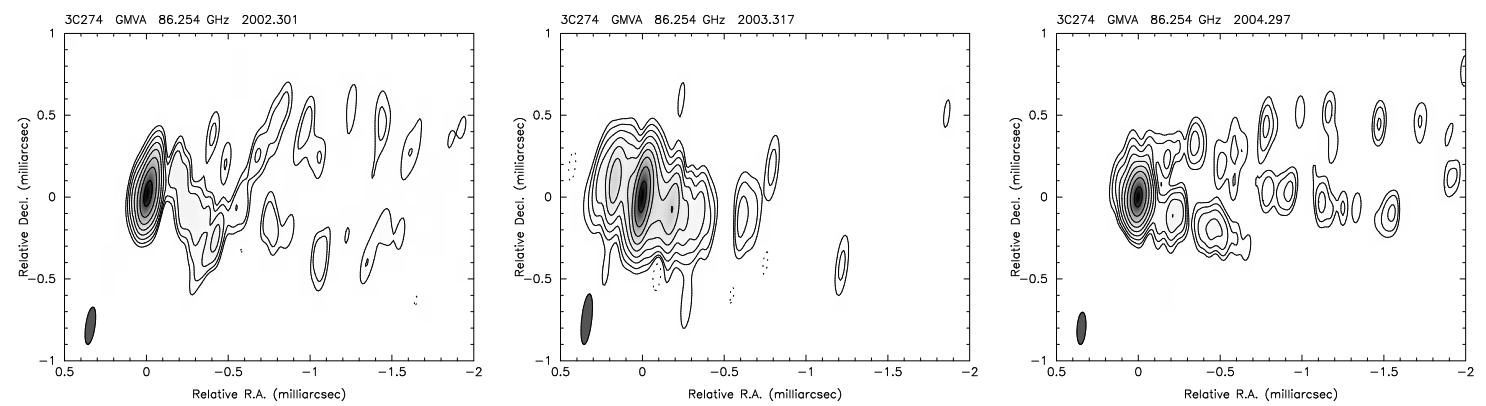

Figure 3: Preliminary 86-GHz VLBI images of M 87 (3C 274) obtained with global 3-mm VLBI in April 2002, 2003, and 2004 (from left to right). The contour levels are at -0.3, 0.3, 0.6, 1.2, 2.4, 4.8, 9.6, 19.2, 38.4, and $76.8 \%$ of the peak flux density of $0.52 \mathrm{Jy} /$ beam (2002), $0.73 \mathrm{Jy} / \mathrm{beam}(2003), 0.35$ $\mathrm{Jy} /$ beam (2004). In the map of 2002, the lowest contour level is omitted. The restoring beam sizes are $0.23 \times 0.057$ mas (2002), $0.31 \times 0.062$ mas (2003), and $0.20 \times 0.054$ mas (2004), respectively.

sensitive millimeter antennas in the Southern Hemisphere, however, will most likely not permit to image Sgr $\mathrm{A}^{*}$ at $1 \mathrm{~mm}$ with sufficiently high dynamic range (and a not too elongated observing beam) for the next $5-10$ years. In this context, the mm-VLBI observations of M 87 are more promising for the search of GR-effects in the vicinity of a SMBH.

Since the mid 1990's, several attempts have been made to obtain a reliable image of M 87 with global 3-mm VLBI. With a correlated flux density of only $\sim 300-600 \mathrm{mJy}$ at the longest $u v$-spacings $(\sim 3 \mathrm{G} \lambda)$, the nucleus of M 87 is relatively weak and fringe detection requires a high sensitivity, which is now provided by the GMVA. The preliminary maps presented in Figure 3 show the structure of the inner jet at three epochs: April 2002, 2003, and 2004, respectively. The observations in 2002 and 2003 were made at $256 \mathrm{Mbit} / \mathrm{s}$, while in 2004 the recording rate was increased to $512 \mathrm{Mbit} / \mathrm{s}$. The amount of visibility data with the three most sensitive telescopes (EB, $\mathrm{PV}, \mathrm{PdB}$ ) largely determines the quality of the maps.

In April 2002, the source was observed with 12 VLBI stations $(8 \times$ VLBA, EB, ON, PV, HSTK). Unfortunately, bad weather at EB and PV limited the dynamic range of the resulting map (Fig. 3, left). Tapering this image with a larger beam (not shown here), however, shows an edgebrightened, transversely resolved and conical jet with similar opening angle as seen in the $43-\mathrm{GHz}$ images of the VLBA ([20], [26]) and also in the later 3-mm map of April 2004 (Figure 3, right).

In April 2003, the source was observed with participation of the phased IRAM interferometer on Plateau de Bure. The combination of this sensitive telescope with the other VLBI stations $(\mathrm{EB}, \mathrm{PV}, \mathrm{ON}, 8 \times \mathrm{VLBA}$ ), resulted in a better map (see Fig. 3, center). The source exhibits a core-jet structure and embedded several distinct emission features aligned at a position angle of $P A \sim-(110-120)^{\circ}$, which coincides with the orientation of the southern border of the jet cone (cf. Fig. 3, right). The two times higher brightness of the VLBI core in this epoch (peak flux densities of $0.52 \mathrm{Jy} /$ beam in $2002,0.73 \mathrm{Jy} /$ beam in 2003 , and $0.35 \mathrm{Jy} /$ beam in 2004) and the dynamic range limitations may explain the lack of faint jet emission, seen at larger core separations in the 2002 and 2004 image. In 2002 and 2004, the jet appeared one-sided with all of the extended jet emission located west of the brightest component. In 2003, however, a partially resolved component located $\sim 0.2$ mas east to the $\sim 4$ times brighter central and more compact component was seen. At present, 
it is unclear if this eastern component should be identified with the jet base (which then must vary in compactness considerably), or if it is part of a counter-jet. More evidence for a counter-jet comes from a new 43-GHz VLBA map obtained by Ly et al., [26], but is not seen at cm-wavelengths ([11]).

In April 2004, M 87 was observed with 10 stations (EB, ON, PV, $7 \times$ VLBA). Due to the failure of its $\mathrm{H}$-maser, the PdB interferometer was not available (a new H-maser is now delivered). Because of the now increased recording rate of $512 \mathrm{Mbit} / \mathrm{s}$, the imaging sensitivity was improved and the source could be reliably imaged. The map in Figure 3 (right) shows again the known Yshaped (conical) jet expansion, most of which takes places on the $\leq 0.3-0.5$ mas scale. Thus, the linear size of the region of jet collimation is $\sim(85-140)$ Schwarzschild radii. The transverse width of the jet at $r=0.5-2$ mas is of the order of $0.5-0.7$ mas, corresponding to $\sim 140-200$ Schwarzschild radii, showing clear signs of edge-brightening and a 'hollow' or at least faint central jet body. If this central jet region would contain a fast electron population (fast spine), the $\geq$ $20-40^{\circ}$ jet inclination relative to the observer could lead to Doppler de-boosting that would reduce its observable brightness. In 2004 (and in 2002) the jet base appeared unresolved, with an upper limit to its size determined by the actual synthesized beam. If we assume that the minor axis of the beam is a measure of the underlying size of the foot-point of the jet, we obtain an upper limit of $54 \mu$ as, corresponding to a spatial scale of only $\leq 15 R_{S}^{9}$. The lower limit of the brightness temperature of the core component is then $T_{B} \geq 2 \cdot 10^{10} \mathrm{~K}$. No firm statement can yet be made with regard to the distance of the jet base relative to the central SMBH. The back-extrapolation of the jet cone to its vertex, however, indicates that this distance is small and of the order of $\leq 0.1-0.3$ mas, or $\leq 30-90 R_{S}^{9}$.

We find it very remarkable that the size of the VLBI core, which should be identified with the region of the jet, where it first becomes radiative, is so small. The existence of a fully developed jet on such small spatial scales will give important new constraints for the theory of jet formation. Jet models based on the magnetic sling shot mechanism (Blandford \& Payne, [7], [8]) assume efficient particle acceleration along the $S$-shaped magnetic field lines up to Lorentz factors of $\gamma=10-20$. The field lines are anchored to the inner part of the rotating accretion disk and probably form helical magnetic flux tubes, which may explain the observed bent and sometimes edge-brightened jet morphology (core-sheath structure). In these models, the diameter of the jet base is defined by the transverse width of the jet after initial acceleration, being of the order $\geq \gamma \cdot 2 R_{L}$, where $\gamma$ is the bulk Lorentz factor and $R_{L}$ is the radius of the light cylinder ([8], [33]). The light cylinder should have a radius of typically $(10-50) R_{S}$ ([[8],[13]). If we assume for the Lorentz factor $2 \leq \gamma \leq 3$ (as derived from the motion seen on pc scales), one would expect a size of the jet base of $\geq(40-300) R_{S}^{9}$, which is at least 3 times larger than the measured size. One way out of the problem is to assume that the central $\mathrm{BH}$ is rotating (Kerr BH). This would reduce the radius of the light cylinder and the width of the jet base by up to a factor of 3 (e.g. [30]). In this case, the observed small size of the VLBI core would favor jet models, in which the jet gains energy directly from BH-rotation ([6], [29], [19]).

Cygnus A: Another argument for the relevance of magnetic acceleration in radio jets comes from multi-frequency studies of the kinematics in the jet and counter-jet of Cygnus A. In Figure $\bigoplus$ (left), we show some VLBI maps of the source at $15-86 \mathrm{GHz}$ (data: Bach et al., see [], [河). At $86 \mathrm{GHz}$, 

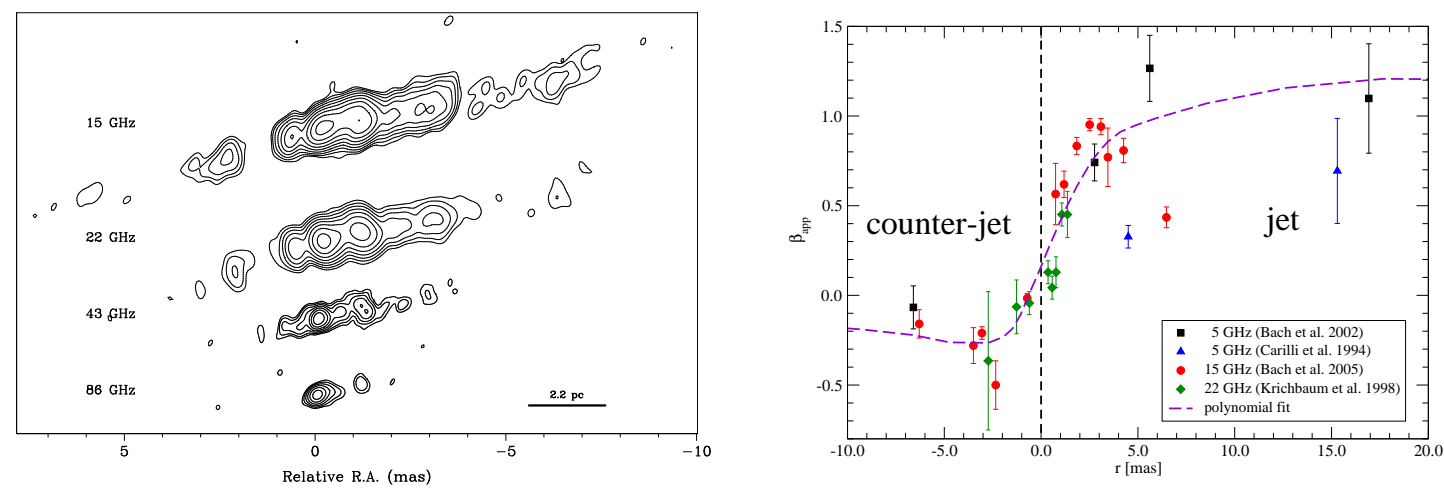

Figure 4: Left: The jet of Cygnus A at four different frequencies: 15, 22, 43, and $86 \mathrm{GHz}$ (top to bottom). The 86-GHz images results from GMVA observations of April 9, 2003 (using 256-Mbps recording). The 15 and 22-GHz observations are phase-referenced. The alignment of the other maps has been done based on morphological similarities. At $86 \mathrm{GHz}$, the beam size is $0.07 \times 0.28 \mathrm{mas}^{2}$ at $P A=-23^{\circ}$, the peak flux density is $0.33 \mathrm{Jy}$ and the rms noise is $5.4 \mathrm{mJy}$. The lowest contours begin at $15 \mathrm{mJy} / \mathrm{beam}$ and increase in steps of 2. Right: Apparent velocity of individual jet components in the jet and counter-jet of Cygnus A plotted versus core separation. New data are combined with data from the literature. The dashed line is a polynomial fit including also data points at larger core separations, which are not shown in the figure. For

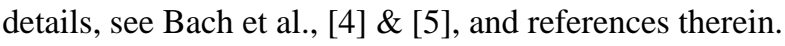

a one-sided core-jet structure is visible, consisting of an unresolved core of $\sim 70 \mu$ as size (linear size $\leq 90$ light-days) and two secondary jet components. Some station failures and a recording rate of only $256 \mathrm{Mbit} / \mathrm{s}$ limited the sensitivity and dynamic range of the map, and prevented the detection of fainter jet and counter-jet emission. We note that the strong frequency dependence of the jet-to-counter-jet ratio suggests the existence of a foreground absorber that attenuates the radiation from the counter-jet ([21]). Clearly, more sensitive mm-VLBI observations are required to detect the counter-jet at a frequency, where the absorber becomes optically thin and the counter-jet becomes brighter. Future high angular resolution images of double-sided jets, like those in Cygnus A, are very important, as they may lead to detection of a gap between the two foot-points of the jet and counter-jet. The size and position of the gap would nicely constrain the position of the central $\mathrm{BH}$, and tell us more about the jet acceleration processes that link the $\mathrm{BH}$ with the jet base. That magnetic acceleration is not just a speculation is demonstrated in Figure $\bigoplus$ (right), showing the measured apparent jet speed as a function of core separation. For an almost perfectly straight jet like that in Cygnus A, jet bending and geometrically caused acceleration (due to differential Doppler boosting) can be neglected. Thus, the observed acceleration must be related to an intrinsic variation of the bulk Lorentz factor. The observed large and systematic variation in apparent jet speed by almost one order of magnitude $(0.1-1) c$ within $\leq 5 \mathrm{pc}$, cannot be easily explained with purely hydrodynamical jet models. Most interesting is the observed shape of the acceleration curve (dashed line in Figure $\bigoplus$ (right)), very nicely reproduced by the model of Vlahakis \& Königl ([35], and Fig. 1e therein) that uses magnetic acceleration in Poynting-dominated jets.

3C 454.3: In AGN, flux density outbursts are usually more pronounced at higher radio frequencies. Quite often, they lead to the ejection/creation of new jet components that subsequently propagate down the jet. One particular advantage of mm-VLBI is the ability to detect these new jet com- 

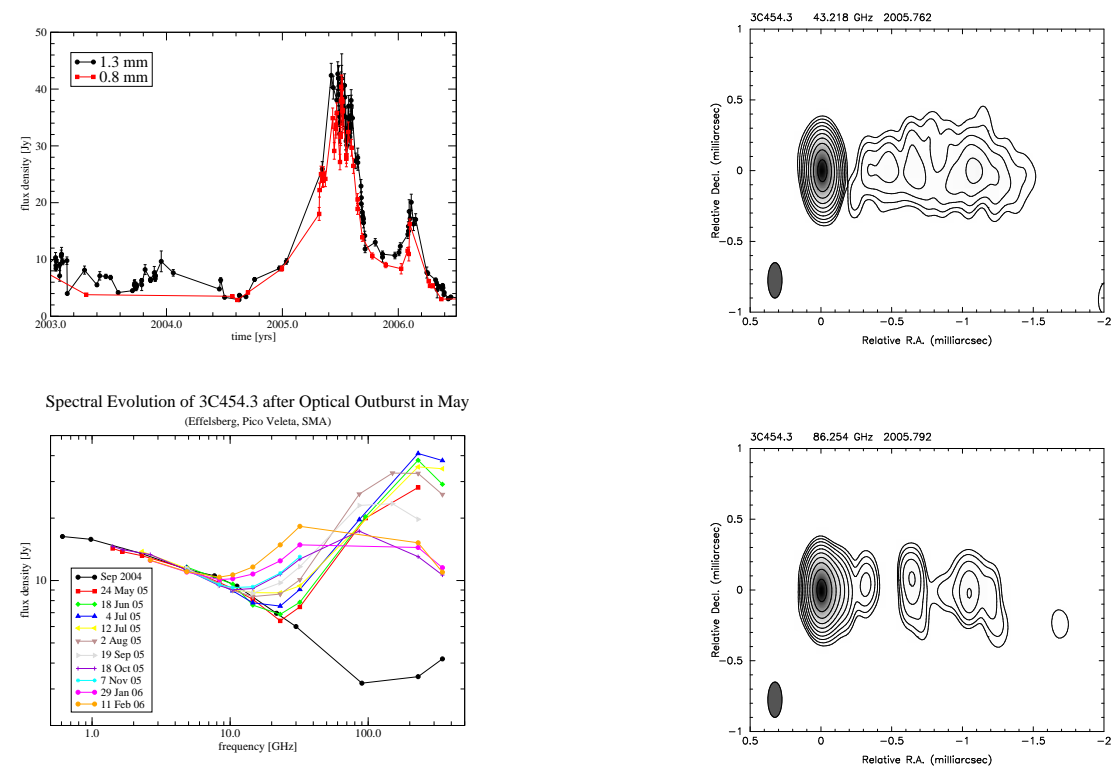

Figure 5: Top Left: The flux-density variability of 3C 454.3 at $1.3 \mathrm{~mm}$ (black) and $0.85 \mathrm{~mm}$ (red). A pronounced flux density peak is seen around epoch 2005.5. The data are from flux-density monitoring performed with the SMA (M. Gurwell, priv. comm.). Bottom Left: Simultaneous radio spectra obtained with a coordinated broad-band spectral monitoring program carried out in 2005-2006. Data from Effelsberg (1-32 GHz), Pico Veleta (90-230 GHz) and from the SMA $(230-345 \mathrm{GHz})$ are combined. For each individual observing date, the measurements were made quasi-simultaneously within less than $1-4$ days. Different symbols and colors denote different observing epochs. Right: Quasi-simultaneous VLBI images of 3C 454.3 at $43 \mathrm{GHz}$ (top, VLBA data: A. Marscher, priv. comm.) and at $86 \mathrm{GHz}$ (bottom, GMVA) of October 6 and 17,2005 , respectively. Both maps are convolved with a common elliptical beam of size $0.25 \times 0.1$ mas. A core elongation observed at the $r=0.1-0.3$ mas scale at $86 \mathrm{GHz}$ is not visible at $43 \mathrm{GHz}$, indicating strong absorption effects in this region of the jet.

ponents in their earliest evolutionary phases and near their origin. In early May 2005, the quasar 3C $454.3(z=0.859)$, showed a large flux density outburst, which was first observed in optical/Xray bands ([14], [16] and references therein) and within one month evolved into a huge millimeterwavelength flare of peak flux density of $\sim 40 \mathrm{Jy}$ at $3 \mathrm{~mm}$ and $1 \mathrm{~mm}$ (Fig. 5, top left). Already during the onset of the radio flare, we started to observe the source with a broad frequency coverage and the aim to monitor the evolution of the radio spectrum. Using the 100-m MPIfR radio telescope we performed a regular flux-density monitoring, rapidly switching between all available receivers (1.4 - $32 \mathrm{GHz}$ ) on each observing date. These data are complemented by quasi-simultaneous measurements made with the 30-m IRAM telescope on Pico Veleta $(90-230 \mathrm{GHz}$, data: H. Ungerechts et al.) and the Sub-millimeter Array (SMA) on Mauna Kea $(230-345 \mathrm{GHz}$, data: M. Gurwell et al.). In Fig. 5 (bottom, left), we show some examples of the resulting broad-band radio spectra, covering a time range from May 2005 to February 2006. For comparison, also a quiescent pre-flare spectrum from RATAN-600 is added (Sep. 2004, black line, data: Trushkin et al., [34]). The figure clearly shows a spectral 'bump', initially peaking near $\sim 230 \mathrm{GHz}$, and then fading and propagating towards longer wavelengths. This behavior appears largely consistent with a moving and expanding relativistic shock, undergoing synchrotron and adiabatic cooling (e.g. [27]). We note that this flare appears very pronounced at short millimeter wavelengths, but at longer wavelengths 


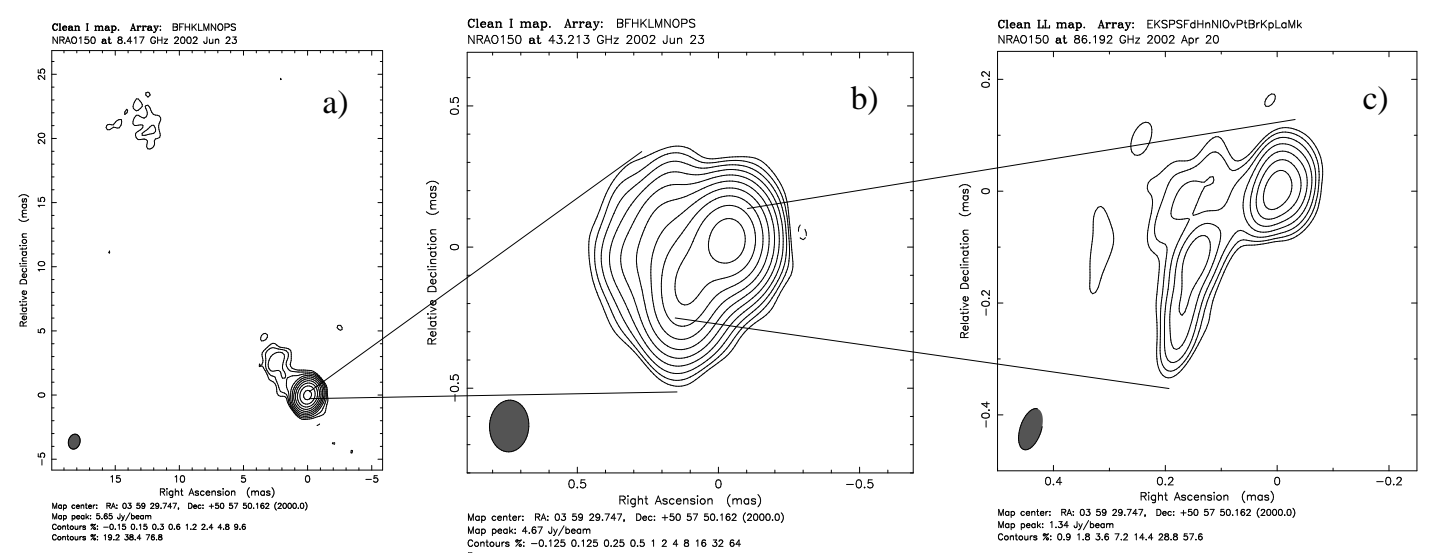

Figure 6: The VLBI jet of NRAO 150. Three maps resulting from observations between April and June 2002 at three different frequencies are shown: $8.4 \mathrm{GHz}$ (a), $43.2 \mathrm{GHz}$ (b), and $86.2 \mathrm{GHz}$ (c). The misalignment and bending of the jet between milliarcsecond and sub-milliarcsecond scales is obvious. Data: Agudo et al., [朋, 级.

(below $\sim 10 \mathrm{GHz}$ ) it did not cause very dramatic variations so far. Therefore, it is expected that most of the flaring and related possible structural variability should occur in the core region that is self-absorbed at cm-wavelengths.

$3 \mathrm{C} 454.3$ has also been monitored with the VLBA and the GMVA by several groups. Unfortunately, no 3-mm VLBI image is yet available at a time near the flare maximum (2005.5). In Figure 5 (right, bottom), we show a 3-mm GMVA image obtained $\sim 3$ months afterwards (Oct. 17, 2005). The position of a new jet component seen at $r=0.1$ mas is consistent with an assumed ejection between 2005.3 and 2005.5 and an apparent speed in the range of $5-14 c$.

For comparison, a 43-GHz VLBA image (data: A. Marscher, priv. comm.) observed 11 days earlier is shown on top of the 3-mm map. Both maps show one-sided inner jet, extending to $r \simeq 1.5$ mas length, with at least three embedded more compact emission components. For the compact, and at $3 \mathrm{~mm}$ slightly elongated core we find a spectral index of $\alpha_{43 / 86 \mathrm{GHz}}=-0.4\left(S \propto v^{\alpha}\right)$. While the $86-\mathrm{GHz}$ map shows two secondary jet components on the $0.8-2.3$ pc scale near the core (at $0.1 \leq r \leq 0.3$ mas), the $43-\mathrm{GHz}$ image does not show similar emission. This could indicate either fast motion within 11 days (of several mas/yr), which is unlikely, or the presence of internal absorption. By integrating the flux in this region, we estimate a highly inverted spectral index in the range of $\alpha_{43 / 86 \mathrm{GHz}}=+1.1 \ldots 2.8$. At a larger core separation, however, the spectrum of the jet is steep again. At present, it is unclear if this spectral inversion is due to a foreground absorber partially covering the inner jet. Future polarization and Faraday rotation measurements could help to answer this question.

NRAO 150: The last important aspect, which we like to address in this paper, is the possibility to study the nature of jet bending and to trace strongly curved jets down to their physical origin with mm-VLBI. In almost all the sources so far imaged with mm-VLBI, the jet curvature increases with decreasing core separation. An exciting example for a curved and possibly 'rotating' jet nozzle is seen in the strong radio source NRAO $150(z \simeq 1.52$, [1]). It shows a long-term light curve 

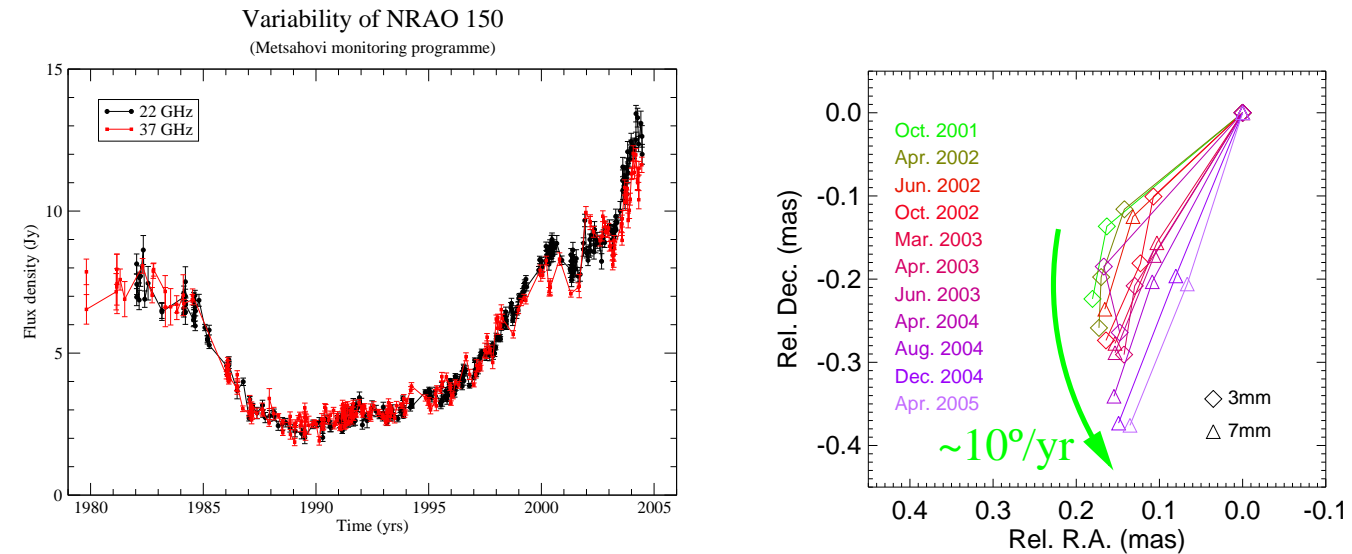

Figure 7: Left: Long term flux density variability of NRAO 150 at $22 \mathrm{GHz}$ (black) and $37 \mathrm{GHz}$ (red). Data are from the Metsähovi monitoring program [32] and from M. Tornikoski (priv. comm.). Right: The orientation of the jet axis of NRAO150 as a function of time. Diamonds (from $86 \mathrm{GHz}$ ) and triangles (from $43 \mathrm{GHz}$ ) mark relative positions of Gaussian model fit components with respect to the VLBI core assumed to be stationary. The lines connecting adjacent jet components illustrate the orientation of the jet ridge-line and its variation with time. Different colors denote various observing epochs (October 2001 to April 2005). Data: Agudo et al. 2005 and in prep..

appearing sinusoidal in the Michigan and Metsähovi flux monitoring programs, and a remarkable flux density increase from $\sim 2 \mathrm{Jy}$ to $\sim 12 \mathrm{Jy}$ at $37 \mathrm{GHz}$ during the last 15 years ([32], see also Fig. 7, left). Motivated by the possibility of a geometrical origin of this variation and a possible periodicity, we began to observe the source with global 3-mm VLBI in the late 1990's (for details see [2]). In Figure 6, we show three VLBI maps obtained at 8, 43, and $86 \mathrm{GHz}$ in spring 2002. At cmwavelengths, a one-sided core-jet structure with a jet extending to at least $r=20$ mas and oriented along $P A \simeq(30-40)^{\circ}$ is seen. At higher angular resolutions, the mm-VLBI images reveal a very different jet orientation of $P A \simeq 140^{\circ}$, showing very pronounced jet bending of $\triangle P A \simeq 110^{\circ}$ within only 0.5 mas $(4.3 \mathrm{pc})$ core separation. Such a strong curvature can be explained by geometrical projection effects, if the inner jet is aligned at a small viewing angle. 43-GHz VLBA and 86-GHz GMVA monitoring observations performed between 1999 and 2005 allowed to follow the motion of the inner-most jet components ( $\beta_{a p p} \simeq 2.7-3.5$ ) and measure the orientation of the axis of the inner jet. In Figure 7, we show a preliminary plot of the positions of the inner jet components as a function of time and aligned with respect to the VLBI core assumed to be stationary. Colored lines connect corresponding components at a given epoch and outline the orientation of the mean jet axis. A systematic clockwise rotation of the mean jet position angle in the plane of the sky towards the south at a rate of $d P A / d t \sim(7-10)^{\circ} / \mathrm{yr}$ is obvious. It is tempting to relate this rotation to the long-term flux density variability, suggesting a timescale of $P \gtrsim 20-25 \mathrm{yrs}$. We note that similar, although less pronounced, jet position angle variations are seen in the pc-scale jets of an increasingly large number of sources (i.e. 3C 84, 3C 120, 0716+714, OJ 287, 3C 273, 3C 345, BL Lac, etc.). This suggests a common physical origin and a fundamental process in AGN radio jets. We stress that the non-ballistic motion of the jet components excludes simple geometrical precession, as is observed in micro-quasars like e.g. SS 433. Whether such 'wobbling' of the jet foot-point is caused by gravitational interaction with another super massive body, by instabilities 

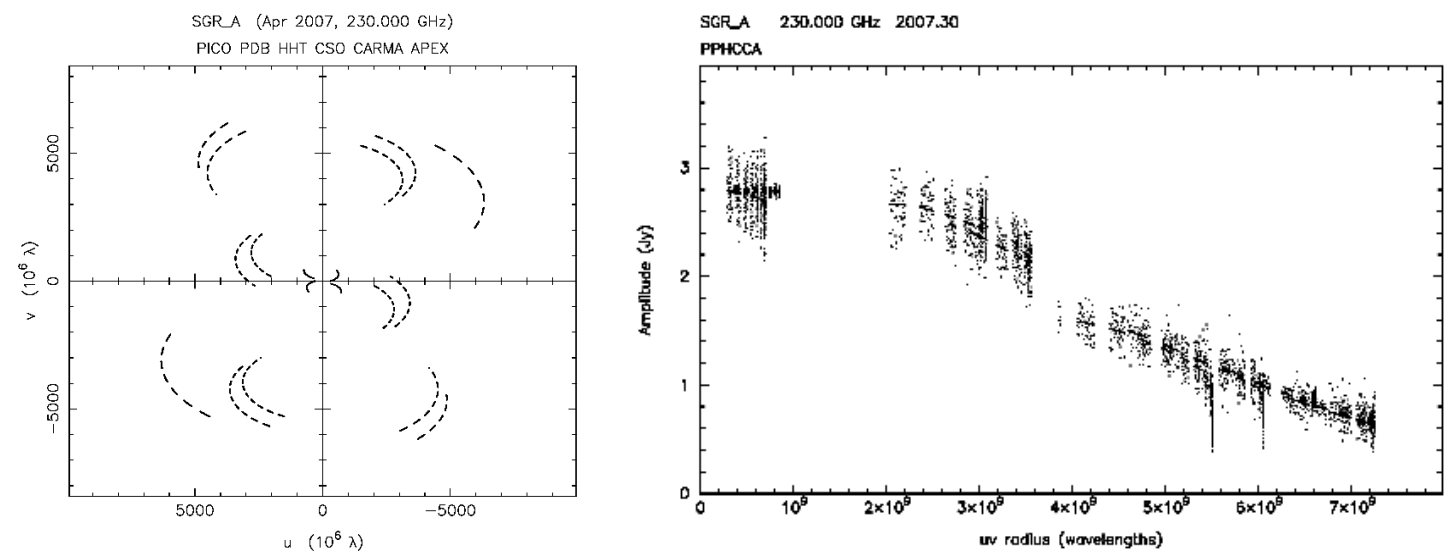

Figure 8: The $u v$-coverage (left) and visibility amplitude plotted versus projected baseline length (right) of a future and not unrealistic global VLBI experiment at $1.3 \mathrm{~mm}(230 \mathrm{GHz})$. For this simulation, the participation of the following stations was assumed: Pico Veleta (30-m, IRAM, Spain), Plateau de Bure $(6 \times 15-\mathrm{m}$, IRAM, France), Heinrich Hertz Telescope (10-m, ARO, Arizona), CSO (10-m, Caltech, Hawaii), CARMA (single 10.4-m, California), and APEX (12-m, MPIfR, Chile). We assumed the brightness distribution as shown in the Figure 9 below.
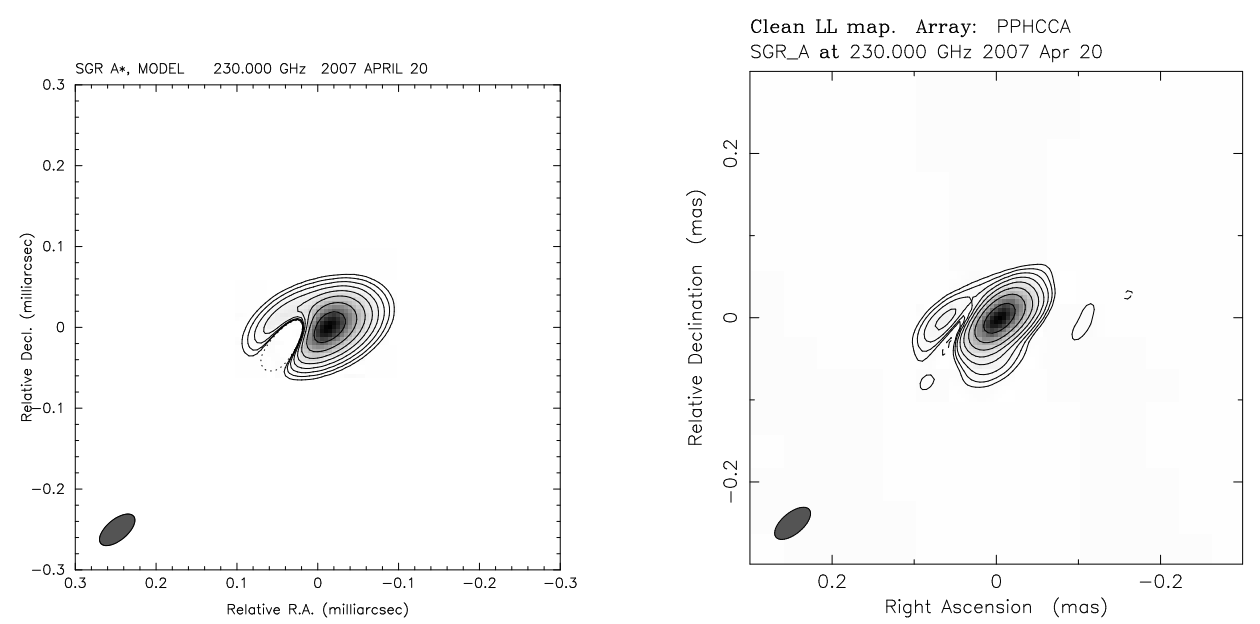

Figure 9: left: Hypothetical brightness distribution of an eccentrically displaced sickle-shaped structure as expected from gravitational light bending around a rotating BH. Contour levels are at $-0.5,0.5,1,2,4,8,16$, 32 , and $64 \%$ of the peak flux density of $1.8 \mathrm{Jy} / \mathrm{beam}$. The model is convolved with a restoring beam of size $50 \times 30 \mu$ as, oriented at $P A=-50^{\circ}$. Right: Reconstructed CLEAN image from the simulated visibility data shown in Figure 8. Contour levels are the same as for the input model. The uniformly weighting restoring beam is $53 \times 26 \mu$ as, oriented at $P A=-50^{\circ}$.

in the accretion disk or at the jet base, or is an inherent property of a $\mathrm{BH}$-accretion disk system (general relativistic effects, e.g. the Lense-Thirring rotation), remains at this point an open question and deserves future studies.

\section{Summary and Outlook}

Global millimeter VLBI provides images of dynamic range of a few hundred and with angular 
resolution of up to $40-50 \mu$ as. Although the sensitivity and the $u v$-coverage are already quite good, there is still room for some improvement. The performance of the VLBA antennas at $86 \mathrm{GHz}$ is still limited by persisting pointing and focusing problems at this high frequency. The lack of a large millimeter antenna in the USA, limits the transatlantic detection threshold to $\geq 50-100 \mathrm{mJy}$ (IRAM-VLBA) and the intra-American baseline sensitivity to $\geq 200-300 \mathrm{mJy}$. The participation of a large telescope like the GBT in 3-mm VLBI would improve the overall array sensitivity by at least a factor of 2 from $\simeq 2 \mathrm{mJy} / \mathrm{h}$ to $\lesssim 1 \mathrm{mJy} / \mathrm{h}$. A similar argument applies for CARMA, when used as a phased array in 3-mm VLBI. A substantial gain in sensitivity comes from a larger observing bandwidth. While the European antennas can now already observe at data rates as high as $1 \mathrm{Gbit} / \mathrm{s}$ (256-MHz bandwidth for 2-bit sampling), the VLBA does not yet support this observing mode. The application of more sophisticated fringe-fitting methods, i.e., by the use of phase corrections from water vapor radiometry (see paper by A. Roy et al., this proceedings) and the enhancement of the AIPS fringe-fitting tasks through more sophisticated incoherent averaging methods ([31]), could further improve the sensitivity of mm-VLBI.

The mm-VLBI imaging of nearby sources (such as Sgr A* and M 87) opens a challenging perspective of being able to study the immediate vicinity of SMBHs with a spatial resolution of only a few to a few ten gravitational radii. Unfortunately, the low declination of these particular sources limits the north-south resolution of the existing mm-VLBI networks. For the imaging of general relativistic effects in nearby black holes, the addition of mm-VLBI capable radio telescopes in the southern hemisphere (South America: APEX, LMT, ALMA, etc. for observations at $\lambda \leq 3 \mathrm{~mm}$ ), but also in Southern Europe (Noto, Yebes, Sardinia Radio Telescope, for observations at $\lambda=7 \& 3 \mathrm{~mm}$ ) and in South Africa (no mm-telescope planned) would be extremely important. To demonstrate this, we show in Figures 8 and 9 a simulation of a future 1.3-mm VLBI experiment with 6 sub-millimeter telescopes: one telescope located in the southern hemisphere (here we used $\operatorname{APEX}(12 \mathrm{~m})$, but other antennas, like the Japanese ASTE $(10 \mathrm{~m})$ or the first ALMA antennas, may be used, if equipped for VLBI). The simulation shows that a possible intrinsic asymmetry in the brightness distribution of Sgr A* could be detected, if one of the aforementioned antennas would be equipped for VLBI (H-maser, VLBI terminal). For this simulation, we assumed state of the art receiver temperatures and a moderate recording rate of $2 \mathrm{Gbit} / \mathrm{s}$ that reflects the ongoing development of the VLBI data acquisition. Even higher data rates (observing bandwidths) are anticipated in the next few years (e.g. Mark5 B, recording at 4 Gbit/s, [37]). The corresponding sensitivity increase will lead to a much larger number of observable objects and to mm-VLBI at even shorter wavelengths $(\lambda \leq 1.3 \mathrm{~mm})$. With an angular resolution of better than $10-20 \mu$ as, one can image compact emission regions of a few gravitational radii size. Similar small scales can be reached with future space VLBI missions at longer wavelengths (e.g. VSOP-2, H. Hirabayashi, this conference) and with X-ray interferometers (e.g. MAXIM, [36]). The combination of high-resolution radio interferometry and interferometric X-ray spectroscopy would indeed form an extremely powerful tool to study SMBHs and their environment with unprecedented accuracy.

\section{Acknowledgments}

We thank the staff of the observatories participating in mm-VLBI, and in particular the telescope and correlator operators and VLBI friends. We thank W. Alef and D. Graham for their help 
in the various stages of data reduction. The VLBI maps presented for 3C 120 have been obtained within a collaboration of some of the authors of this paper with J.L. Gómez and A. Marscher. The VLBI results presented for 3C 454.3 have been obtained partly within a larger collaboration of some of the authors with Boston University Group (A. Marscher et al.), the Finnish group at Tuorla Observatory (T. Savolainen, K. Wiik, et al.), and IRAM (H. Ungerechts, H. Wiesemeyer, C. Thum et al.). Especially, we would like to thank A. Marscher for providing data prior to publication. For the use of their partly unpublished flux density monitoring data, we thank M. Gurwell (SMA), H. Ungerechts (IRAM), and M. Tornikoski (MRO). I. Agudo acknowledges financial support from the European Commission for Science and Research through the ENIGMA network (contract HPRNCT-2002-00321).

\section{References}

[1] Acosta-Pulido, J.A., et al., 2007, A\&A, in preparation.

[2] Agudo, I., Krichbaum, T.P., Bach, U., et al., 2005, Rev. Mex. AA, in press, (astro-ph/0511268).

[3] Agudo, I., et al., 2007, in preparation.

[4] U. Bach, T.P.Krichbaum, E. Middelberg, et al., 2004, in: Proceedings of the 7th EVN Symposium, ed. R. Bachiller et al., OAN, Madrid, p. 155.

[5] U. Bach, M. Kadler, T.P.Krichbaum, et al., 2005, in: Future Directions in High Resolution Astronomy ASP Conference Proceedings, Vol. 340. Edited by J. Romney and M. Reid. San Francisco: Astronomical Society of the Pacific, p. 30.

[6] Blandford, R.D., \& Znajek, R.L., 1977, MN 179, 433.

[7] Blandford, R.D., \& Payne, D.G., 1982, MN 199, 883.

[8] Camenzind, M., 1990, RvMA 3, 234.

[9] Caproni, A., \& Abraham, Z., 2004, MN 349, 1218.

[10] De Villiers, J.P., Hawley, J.F., \& Krolik, J.A., 2003, ApJ 599, 1238.

[11] Dodson, R., Edwards, P.G., \& Hirabayashi, H., 2006, PASJ 58, 243.

[12] Doeleman, S.S., Phillips, R.B., Rogers, A.E.E., et al., 2005, in: Future Directions in High Resolution Astronomy: The 10th Anniversary of the VLBA, ASP Conf. Proc. Vol. 340, ed. J. Romney \& M. Reid, p.605

[13] Fendt, C., \& Memola, E., 2001, A\&A 365, 631.

[14] Fuhrmann, L., Cucchiara, A., Marchilli, N., et al., 2006, A\&A 445, 1.

[15] Gammie, C.F., McKinney, J.F., \& Toth, G., 2003, ApJ 589, 444.

[16] Giommi, P., Blustin, A.J., Capalbi, M, et al., 2006, A\&A, 456, 911.

[17] Gómez, J.L., Marscher, A.P., Alberdi, A., Jorstad, S.G., \& Agudo, I., 2001, ApJ 561, L161.

[18] Hardee, P.E, Walker, R.C., \& Gómez, J.L., et al., 2005, ApJ 620, 646.

[19] Hawley, J.F., \& Krolik, J.H., ApJ, 641, 103.

[20] Junor W., Biretta J.A., Livio M., 1999, NAT 401, 891. 
[21] Krichbaum, T.P., Alef, W., Witzel. A., et al., 1998, A\&A 329, 873.

[22] Krichbaum, T.P., Graham, D.A., Witzel, A., et al., 1998, A\&A 335, 106.

[23] Krichbaum, T.P., Graham, D.A., Alef, W., et al., 2002, in: 6th EVN Conf., ed. E. Ros et al., p.125 (astro-ph/0207022).

[24] Krichbaum, T.P., Graham, D.A., Alef, W., et al., 2004, in: Proceedings of the 7th EVN Symposium, ed. R. Bachiller et al., OAN, Madrid, p.15 (astro-ph/0411487).

[25] Krichbaum, T.P., Graham, D.A, Bremer, M., et al., in: Galactic Center Workshop 2006 - "From the Center of the Milky Way to Nearby Low-Luminosity Galactic Nuclei", ed. R. Schödel, G. Bower, et al., 2007, Journal of Physics: Conference Series by Institute of Physics Publishing, Bad Honnef, in press (astro-ph/0607072).

[26] Ly, C., Walker, R.C., \& Wrobel, J.M., 2004, AJ 127, 119.

[27] Marscher, A.P., \& Gear, W.K., 1985, ApJ 298, 114.

[28] Marscher, A.P., Jorstad, S.G., Gómez, J.L., McHardy, I.M., Krichbaum, T.P., \& Agudo, I., 2007, ApJ, submitted.

[29] McKinney J.C., 2006, MN 368, 1561.

[30] Meier D.L., Koide S., \& Uchida Y., 2001, Sci 291, 84.

[31] Rogers, A.E.E., Doeleman, S.S., \& Moran, J.M., 1995, AJ 109, 1391.

[32] Teräsranta, H. Wiren, S, Koivisto, P., et al., 2005, A\&A 440, 409.

[33] Tomimatsu A., \& Takahashi M., 2003, ApJ 592, 321.

[34] Trushkin, S.A., Harinov, M.A., Michailov, A.G., 2005, Astro. Telegram 488, May 12.

[35] Vlahakis, N., \& Königl, A., 2004, ApJ 605, 656.

[36] White, N., 2000, NAT 407, 146.

[37] Whitney, A., 2006, The Mark 5B VLBI Data System, in: IVS 2006 General Meeting Proceedings, eds. D. Behrend \& K. Baver, in press. 